\title{
Texture Analysis Based on Gd-EOB-DTPA- Enhanced MRI for Identifying Vessels Encapsulating Tumor Clusters (VETC)-Positive Hepatocellular Carcinoma
}

\author{
Yanfen $\operatorname{Fan}^{1,2, *}$ \\ Yixing $\mathrm{Yu}^{\mathrm{l}, 2, *}$ \\ Ximing Wang ${ }^{1,2}$ \\ Mengjie $\mathrm{Hu}^{1,2}$ \\ Mingzhan $\mathrm{Du}^{3}$ \\ Lingchuan Guo ${ }^{3}$ \\ Shifang Sun ${ }^{4}$ \\ Chunhong $\mathrm{Hu}^{1,2}$ \\ 'Department of Radiology, The First \\ Affiliated Hospital of Soochow University, \\ Suzhou, Jiangsu, 215006, People's \\ Republic of China; ${ }^{2}$ Institute of Medical \\ Imaging of Soochow University, Suzhou, \\ Jiangsu, 215006, People's Republic of \\ China; ${ }^{3}$ Department of Pathology, The \\ First Affiliated Hospital of Soochow \\ University, Suzhou, Jiangsu, 215006, \\ People's Republic of China; ${ }^{4}$ School of \\ Mechatronic Engineering and \\ Automation, Shanghai University, \\ Shanghai, 200444, People's Republic of \\ China
}

*These authors contributed equally to this work

\begin{abstract}
Purpose: To determine the potential findings associated with vessels encapsulating tumor clusters (VETC)-positive hepatocellular carcinoma (HCC), with particular emphasis on texture analysis based on gadolinium-ethoxybenzyl-diethylenetriamine pentaacetic acid (GdEOB-DTPA)-enhanced MRI.
\end{abstract}

Methods: Eighty-one patients with VETC-negative HCC and 52 patients with VETC-positive HCC who underwent Gd-EOB-DTPA-enhanced MRI before curative partial hepatectomy were retrospectively evaluated in our institution. MRI texture analysis was performed on arterial phase (AP) and hepatobiliary phase (HBP) images. The least absolute shrinkage and selection operator (LASSO) logistic regression was used to select texture features most useful for identifying VETC-positive HCC. Univariate and multivariate analyses were used to determine significant variables for identifying the VETC-positive HCC in clinical factors and the texture features of MRI. Receiver operating characteristic (ROC) analysis and DeLong test were performed to compare the identified performances of significant variables for identifying VETC-positive HCC.

Results: LASSO logistic regression selected 3 features in AP and HBP images, respectively. In multivariate analysis, the Log-sigma-4.0-mm-3D first-order Kurtosis derived from AP images (odds ratio $[\mathrm{OR}]=4.128, P=0.001$ ) and the Wavelet-LHL-GLDM Dependence Non Uniformity Normalized derived from HBP images ( $\mathrm{OR}=2.280, P=0.004)$ were independent significant variables associated with VETC-positive HCC. The combination of the two texture features for identifying VETC-positive HCC achieved an AUC value of 0.844 (95\% confidence interval CI, $0.777,0.910)$ with a sensitivity of $80.8 \%(95 \% \mathrm{CI}, 70.1 \%, 91.5 \%)$ and specificity of $74.1 \%$ (95\% CI, 64.5\%, 83.6\%).

Conclusion: Texture analysis based on Gd-EOB-DTPA-enhanced MRI can help identify VETC-positive HCC.

Keywords: hepatocellular carcinoma, texture analysis, GD-EOB-DTPA, quantitative, VETC

\section{Introduction}

Global Cancer Statistics 2018 reported about 841,000 new cases and 782,000 deaths annually for liver cancers. ${ }^{1}$ Hepatocellular carcinoma (HCC) accounts for $75 \%-90 \%$ of primary liver cancers and constitutes a major global health problem. ${ }^{1-3}$ For the therapeutic strategies, partial hepatectomy remains the most effective measure for resectable HCCs with preserved liver function. ${ }^{3-5}$ However, after curative resection,
Department of Radiology, The First

Affiliated Hospital of Soochow University,

Suzhou, Jiangsu, 2 I5006, People's Republic of China

Tel +86 I3506219750

Email sdhuchunhong@sina.com 
the recurrence rate was more than $50 \%$, even approximately $70 \%$ at five years. ${ }^{6,7}$ The frequent relapse is mainly due to early metastasis. ${ }^{8}$ Vessels encapsulating tumor clusters (VETC), a histological vascular pattern, has been identified as a predictor of micro-metastasis in HCC after resection. ${ }^{9}$ Recently, Renne et al stated that the VETC was a powerful predictor of aggressive HCC. ${ }^{10}$ It was associated with poor prognosis after partial hepatectomy for HCC, the presence of VETC was associated with early recurrence, poor overall survival (OS) and shorter disease-free survival (DFS). ${ }^{9,10}$ Moreover, Fang et $\mathrm{al}^{8}$ and Sugino et $\mathrm{al}^{11,12}$ demonstrated the regulatory mechanisms involved in the metastasis of VETC-positive HCC, a novel vascular pattern promotes metastasis of HCC in an epithelial-mesenchymal transitionindependent manner, which were associated with specific biological activities and levels of gene expression (highly expressed or lowly expressed). ${ }^{10,13}$ Then, Fang et al also showed that VETC is a predictor of Sorafenib benefit in patients with HCC. ${ }^{14}$ Although other histopathological markers such as tumor differentiated degree and microvascular invasion of HCC are widely validated and used in clinical practice to predict the prognosis and recurrence, ${ }^{15-17}$ identification of VETC status could help select HCC patients with poor prognosis, further for the development of antimetastasis (anti-angiogenesis) therapy or molecularly targeted drugs treatment in the future.

However, VETC is a histologic finding whose diagnosis can only be gotten post-operatively. Accordingly, the preoperative diagnosis of VETC status in patients with HCC is essential to help decide therapeutic strategies. Recently, our group has revealed that qualitative imaging features (non-rim diffuse and heterogeneous arterial phase hyperenhancement) of Gd-EOB-DTPA-enhanced MRI was helpful to predict VETC-positive HCCs. ${ }^{18}$ Rhee et al ${ }^{19}$ and Mulé et $\mathrm{al}^{20}$ have also revealed that an arterial phase hypovascular component of Gd-EOB-DTPA-enhanced MRI and substantial necrosis of multiphase contrastenhanced MRI could help identify macrotrabecularmassive HCC, which have similar pathological figures to partial VETC-positive HCC. Although previous studies have revealed the risk factors, determinations of the imaging characteristics of HCC were usually inconsistent, as qualitative imaging characteristics of MRI were subjective, which were determined by radiologists, who can vary by differences in interpretation, personal preference, and experience level.

Texture analysis recently provides an objective, quantitative assessment of tumor heterogeneity by analyzing the distribution and relationship of pixel or voxel grey levels in the images and could reflect information on the lesion microenvironment. ${ }^{21-23}$ Accordingly, we speculate it is a promising imaging approach for identifying the VETC-positive HCC.

To our best knowledge, there has been no study applying texture analysis based on GD-EOB-DTPA-enhanced MRI to identify the VETC-positive HCC. This study aimed to investigate potential MRI texture characteristics associated with VETC-positive HCC.

\section{Materials and Methods \\ Patients}

The procedures in this study were compliance with the Declaration of Helsinki. The Institutional Review Board (IRB) of The First Affiliated Hospital of Soochow University reviewed and approved this retrospective study. The informed consent from patient was waived, as patient consent to review their medical records was not required by the IRB. Patients' privacy was ensured, and the data were anonymized or maintained with confidentiality. From January 2016 to October 2020, patients with a postoperative pathological diagnosis of $\mathrm{HCC}$ in our single institution were consecutively enrolled for this study. The inclusion criteria were as follows: (1) have received Gd-EOB-DTPA-enhanced MRI of the liver within one month before surgery; (2) immunochemical staining for a cluster of differentiation 34 (CD34); (3) if multiple lesions were present, we included the largest one with its matched immunohistochemical diagnosis. The exclusion criteria were as follows: (1) received previous treatment, such as anti-tumor therapies, radiofrequency ablation, transcatheter arterial chemoembolization (TACE), and so on. (2) incomplete clinical data; (3) inadequate specimens for histological analysis (necrosis $>$ $90 \%$ ); (4) images with noticeable artifacts.

\section{Evaluation of VETC}

Two experienced pathologists (pathologist \#1 and pathologist \#2, with more than 15 and 8 years of experience in abdominal pathology, respectively) evaluated all available histological slides independently. Occasional inconsistencies were discussed to reach a consensus. Both of the two pathologists blinded to the clinical, laboratory, and imaging results of the patients.

The VETC was defined as the presence of vessels that formed cobweb-like networks, encapsulated and separated 
individual tumor clusters with an unequivocal and continuous lining of CD34 positive endothelium. ${ }^{8,10}$ Referring to previous studies, ${ }^{8-10}$ we evaluated the VETC index from $0 \%$ to $100 \%$ in $5 \%$ of the units in this study, and defined VETC-positive HCC as VETC index $\geq 5 \%$ by CD34 immunostaining.

\section{MRI Acquisition Sequence}

All MR images were acquired using a 3.0-T MRI scanner (Magnetom Verio; Siemens Healthcare, Erlangen, Germany) with a 16-channel phase-array coil covering the whole liver. The MR acquisition sequence included: 1) three-dimensional volume interpolated breath-hold examination (3D VIBE) T1weighted in-phase and out-phase axial imaging; 2) a respiration-triggered turbo spin-echo (TSE) T2-weighted fat-saturation (FS) axial and coronal imaging; 3) a freebreathing single-shot echo-planar diffusion-weighted imaging (DWI, $\mathrm{b}=50,800 \mathrm{sec} / \mathrm{mm}^{2}$ ) axial imaging; 4) The dynamic enhancement was also performed with the 3D VIBE T1weighted FS sequence axial and coronal imaging. The detailed information of each scan sequence was shown in Table 1.

All patients needed a food-fast of more than 6 hours and a water-fast of more than 4 hours before scanning. A dose of $25 \mu \mathrm{mol}$ per $\mathrm{kg}$ of body weight of GdEOB-DTPA (Primovist ${ }^{\circledR}$, Bayer Schering Pharma, AG, Berlin, Germany) was administered bolus rapidly. It was immediately followed by $30 \mathrm{~mL}$ saline at a rate of $1 \mathrm{~mL} / \mathrm{s}$. The arterial phases (AP), portal venous phases (PVP), and transitional phase (TP) images were obtained during suspended respiration at 30-35 sec, 65-70 sec, and 180-185 sec, respectively. The HBP images were obtained at 20 min after the injection of Gd-EOB-DTPA. We retrieved the AP and HBP images from a picture archiving and communication system (PACS, Neusoft Version 5.5).

\section{MRI Texture Analysis}

MRI texture analyses include tumor segmentation, image pre-processing, feature extraction, feature selection, and MRI texture analysis. Radiologists manually segmented tumors on the AP and HBP images with 3D Slicer (http://www.slicer.org). ${ }^{24,25} \mathrm{~A}$ three-dimensional (3D) region of interest (ROI) covering the whole tumor was delineated along the edge of tumors. Two radiologists (radiologist \#1, 5 years of liver imaging experience; radiologist \#2, 8 years of liver imaging experience) were involved in tumor segmentation. To assess interobserver reproducibility, radiologist $\# 1$ and radiologist \#2 independently segment 30 randomly chosen patients with HCC. To assess intra-observer reproducibility, radiologists \#1 repeated tumor segmentation on the same 30 randomly selected patients once, at another day in one week. The remaining image segmentation was independently completed by radiologist \#1.

Image pre-processing was performed using the wavelet and Laplacian of Gaussian (LoG) filters, bin discretization referring to image biomarker standardization initiative (IBIS). ${ }^{26}$ Features were extracted from each 3D ROI using the Slicer Radiomics extension incorporating the pyradiomics library into $3 \mathrm{D}$ Slicer. $^{24}$ For each unique tumor segmentation, extracted features included firstorder statistics, shape and texture features, which were gray level co-occurrence matrix (GLCM), gray level size zone matrix (GLSZM), gray level run length matrix (GLRLM), gray level dependence matrix (GLDM) and neighboring gray tone dependence matrix (NGTDM).

The reproducibility analysis was performed by using the inter-class and intra-class correlation coefficient (ICC). Features with both intra-observer and inter-observer ICC values greater than 0.8 (indicating excellent stability) were selected for subsequent LASSO logistic regression analysis. $^{27}$ By optimizing the tuning parameter $(\lambda)$ in LASSO regression with five-fold cross-validation, the features with non-zero coefficients were selected for further MRI texture analysis. ${ }^{28}$ The flowchart is shown in Figure 1.

\section{Statistical Analysis}

Continuous variables were compared using the MannWhitney U nonparametric rank-sum test. Categorical variables were compared using the $\chi^{2}$ test. Variables

Table I MRI Scan Sequence and Parameters

\begin{tabular}{|l|c|c|c|c|}
\hline Parameter & $\begin{array}{c}\text { TIW IP } \\
\text { and OP } \\
\text { Imaging }\end{array}$ & $\begin{array}{c}\text { TIW } \\
\text { VIBE FS } \\
\text { Imaging }\end{array}$ & $\begin{array}{c}\text { T2W FS } \\
\text { Imaging }\end{array}$ & DWI \\
\hline $\begin{array}{l}\text { Repetition time } \\
(\mathrm{ms})\end{array}$ & 4.16 & 3.9 & 4098 & 5300 \\
\hline $\begin{array}{l}\text { Echo time } \\
(\mathrm{ms})\end{array}$ & 2.58 and I.35 & 1.89 & 105 & 57 \\
\hline $\begin{array}{l}\text { Field of view } \\
\left.(\mathrm{mm})^{2}\right)\end{array}$ & $260 \times 320$ & $260 \times 320$ & $260 \times 320$ & $260 \times 300$ \\
\hline Matrix & $380 \times 320$ & $380 \times 320$ & $400 \times 320$ & $192 \times 400$ \\
\hline $\begin{array}{l}\text { Section thickness } \\
(\mathrm{mm})\end{array}$ & 5 & 3 & 6 & 8 \\
\hline
\end{tabular}

Abbreviations: TIW, TI weighted; IP, in-phase; OP, opposed-phase; VIBE, volumetric interpolated breath hold examination; FS, fat suppression; DWI, diffusion weighted imaging. 


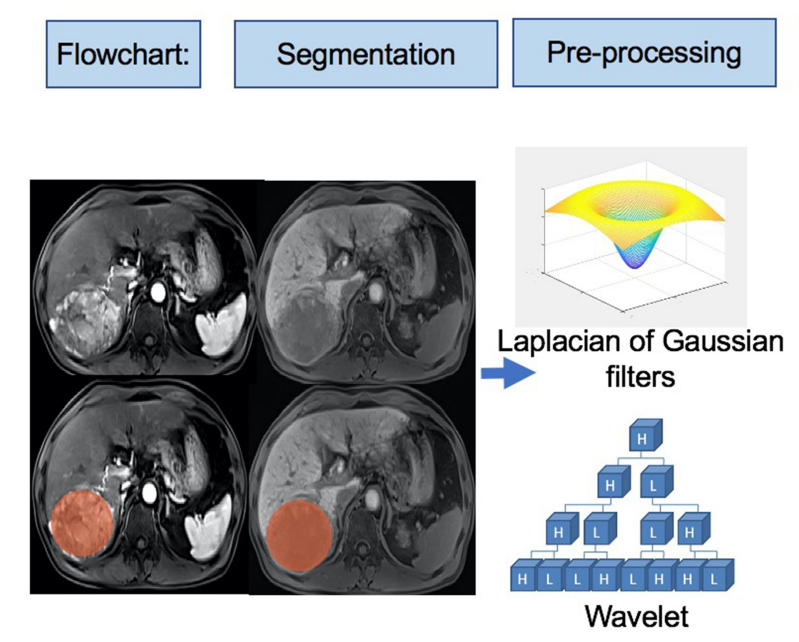

Figure I Flowchart of texture analysis.

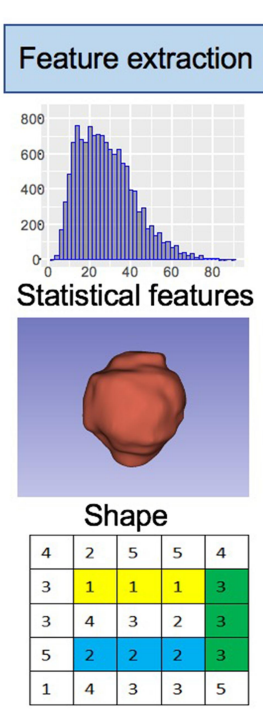

Texture features

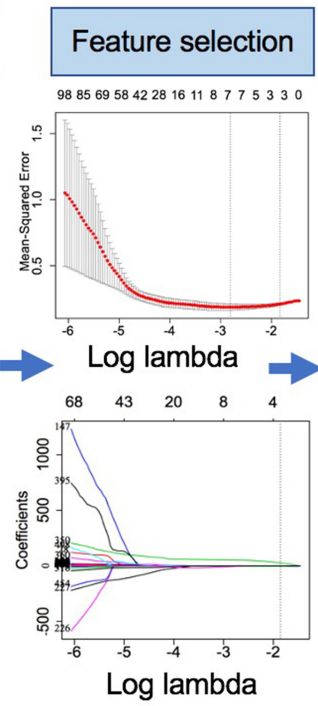

Feature analysis
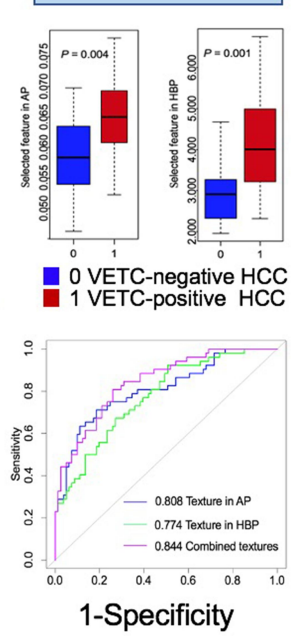

significantly associated with VETC-positive HCC by univariate analysis were considered as candidates for backward stepwise multivariate analysis. The backward stepwise selection with Akaike's information criterion (AIC) as the stopping rule was applied to perform multivariate logistical analysis. The Hosmer-Lemeshow test was conducted to explain the goodness of fit of the multivariate logistic model. ROC analysis of the significant findings was performed to evaluate their identified performance; AUC, sensitivity, specificity, positive likelihood ratio, and negative likelihood ratio with $95 \%$ confidence intervals were calculated. AUC values were compared using the DeLong test. All tests were two-sided, and $P<$ 0.05 was considered significant.

Statistical analysis was performed using the $\mathrm{R}$ software (version 3.6.3, R Project for Statistical Computing, http://www.r-project.org). LASSO and multivariable logistic regression analyses were performed using the "glmnet" and "rms" packages. ROC analysis and DeLong test were performed using the "reportROC" and "pROC" packages. The HosmerLemeshow test was conducted with the "generalhoslem" package.

\section{Results}

\section{Baseline Characteristics of Patients}

Overall, 133 patients (108 men and 25 women; median age 60 years, range $30-86$ years) were included in this study,
81 patients with VETC-negative HCCs and 52 patients with VETC-positive HCCs. The baseline characteristics of 133 patients are shown in Table 2. The higher serum AFP ( $>400 \mathrm{U} / \mathrm{L})$ and AST ( $>40 \mathrm{U} / \mathrm{L}$ ) levels were more frequently observed in the VETC-positive group than that in the VETC-negative HCC group $(P=0.026$ vs $P=$ 0.003) (Tables 2 and 3). In VETC-positive HCC group, the median (IQR) of tumor maximum diameter was 6.210 $(4.102,9.644) \mathrm{cm}$; in VETC-negative HCC group, the median (IQR) of tumor maximum diameter was 3.143 $(2.139,4.504) \mathrm{cm}(P<0.001)$. The greater maximum diameter $(>5 \mathrm{~cm})$ of $\mathrm{HCC}$ was more frequently observed in the VETC-positive group than that in the VETCnegative HCC group $(P<0.001)$ (Tables 2 and 3). There were no significant differences in the gender, age, history of hepatitis, cirrhosis, serum GGT levels, and multiplicity between the VETC-positive group and VETC-negative group (all $P>0.05$ ) (Table 2).

\section{Reproducibility and Texture Selection}

A total of 1316 features were extracted from the AP and HBP images, respectively. For AP images texture analysis, there were 555 features with the inter-observer ICC value $\geq 0.8$ and 534 features with the intraobserver ICC value $\geq 0.8$; for HBP images texture analysis, there were 600 features with the interobserver ICC value $\geq 0.8$ and 613 features with the inter-observer ICC value $\geq 0.8$. Finally, 486 and 512 
Table 2 Clinical Characteristics of Patients with VETC-Positive HCC and VETC-Negative HCC

\begin{tabular}{|c|c|c|c|}
\hline Variables & VETC-Negative Group $(n=8 I)$ & VETC-Positive Group $(n=52)$ & $P$ value \\
\hline Age (median [IQR]) & $61.00[50.00,66.00]$ & $58.00[51.00,67.25]$ & 0.810 \\
\hline Gender (\%) & & & 0.901 \\
\hline Male & $65(80.2)$ & $43(82.7)$ & \\
\hline Female & $16(19.8)$ & $9(17.3)$ & \\
\hline Hepatitis (\%) & & & 0.542 \\
\hline $\mathrm{HBV}$ or/and HCV & $69(85.2)$ & $47(90.4)$ & \\
\hline Negative & $12(14.8)$ & $5(9.6)$ & \\
\hline \multicolumn{4}{|l|}{ Cirrhosis (\%) } \\
\hline Present & $49(60.5)$ & $33(63.5)$ & \\
\hline Absent & $32(39.5)$ & $19(36.5)$ & 0.872 \\
\hline ALT (\%) & & & 0.059 \\
\hline$>50 \mathrm{U} / \mathrm{L}$ & $15(18.5)$ & $18(34.6)$ & \\
\hline$\leq 50 \mathrm{U} / \mathrm{L}$ & $66(81.5)$ & $34(65.4)$ & \\
\hline AST (\%) & & & 0.003 \\
\hline$>40 \mathrm{U} / \mathrm{L}$ & $19(23.5)$ & $26(50.0)$ & \\
\hline$\leq 40 \mathrm{U} / \mathrm{L}$ & $62(76.5)$ & $26(50.0)$ & \\
\hline GGT (\%) & & & 0.302 \\
\hline$>60 \mathrm{U} / \mathrm{L}$ & $36(45.0)$ & $29(55.8)$ & \\
\hline$\leq 60 \mathrm{U} / \mathrm{L}$ & $44(55.0)$ & $23(44.2)$ & \\
\hline AFP (\%) & & & 0.026 \\
\hline$>400 \mathrm{U} / \mathrm{L}$ & $17(2 \mid .0)$ & $21(40.4)$ & \\
\hline$\leq 400 \mathrm{U} / \mathrm{L}$ & $64(79.0)$ & $31(59.6)$ & \\
\hline Multiplicity (\%) & $69(85.2)$ & $39(75.0)$ & 0.215 \\
\hline$\geq 2$ & $69(85.2)$ & $47(90.4)$ & \\
\hline 1 & $12(14.8)$ & $13(25.0)$ & \\
\hline Maximum diameter (\%) & & & $<0.001$ \\
\hline$>5 \mathrm{~cm}$ & $17(2 \mid .0)$ & $31(59.6)$ & \\
\hline$\leq 5 \mathrm{~cm}$ & $64(79.0)$ & $21(40.4)$ & \\
\hline $\begin{array}{l}\text { Maximum diameter } \\
(\text { median }[\mathrm{IQR}]) \mathrm{cm}\end{array}$ & $3.143(2.139,4.504)$ & $6.210(4.102,9.644)$ & $<0.001$ \\
\hline
\end{tabular}

Abbreviations: AFP, alpha-fetoprotein; ALT, alanine aminotransferase; AST, aspartate aminotransferase; GGT, gamma-glutamyl transferase; HBV, hepatitis B virus; HCV, hepatitis C virus; IQR, interquartile range.

features in AP and HBP images with both inter-observer ICC and intra-observer ICC values greater than 0.8 were selected for subsequent LASSO analysis. In the 133 patients, the further LASSO analysis selected the 3 most useful features based on Gd-EOB-DTPAenhanced MRI in the AP and HBP images, respectively. 
Table 3 Univariate and Multivariate Analyses in Identifying VETC-Positive HCC

\begin{tabular}{|c|c|c|c|c|}
\hline \multirow[t]{2}{*}{ Variables } & \multicolumn{2}{|c|}{ Univariate Analysis } & \multicolumn{2}{|c|}{ Multivariate Analysis } \\
\hline & OR & $P$ value & OR & $P$ value \\
\hline \multicolumn{5}{|l|}{ Clinical factors } \\
\hline AFP & 1.529 & 0.017 & & \\
\hline AST & $\mathrm{I} .754$ & 0.002 & & \\
\hline Maximum diameter & 3.211 & $<0.001$ & & \\
\hline \multicolumn{5}{|l|}{ AP images texture feature } \\
\hline $\begin{array}{l}\text { Original Shape } \\
\text { Least Axis Length }\end{array}$ & 3.760 & $<0.001$ & & \\
\hline $\begin{array}{l}\text { Log-sigma-4.0-mm-3D } \\
\text { first-order Kurtosis }\end{array}$ & 7.355 & $<0.001$ & 4.128 & 0.001 \\
\hline Wavelet-HHL GLSZM Entropy & 3.422 & $<0.001$ & & \\
\hline \multicolumn{5}{|l|}{ HBP images texture feature } \\
\hline Wavelet-LLL GLCM ImcI & 0.287 & $<0.001$ & & \\
\hline $\begin{array}{l}\text { Log-sigma-I.0-mm-3D GLSZM } \\
\text { Gray Level Non Uniformity }\end{array}$ & 5.177 & $<0.001$ & & \\
\hline $\begin{array}{l}\text { Wavelet-LHL GLDM } \\
\text { Dependence Non Uniformity Normalized }\end{array}$ & 3.470 & $<0.001$ & 2.280 & 0.004 \\
\hline
\end{tabular}

Abbreviations: AFP, alpha-fetoprotein; AST, aspartate aminotransferase; AP, arterial phase; HBP, hepatobiliary phase; GLCM, gray level co-occurrence matrix; GLDM; gray level dependence matrix; GLSZM, gray level size zone matrix; Imcl, informational measure of correlation I; HHL, highpass filter, highpass filter, lowpass filter; LLL; lowpass filter, lowpass filter, lowpass filter; LHL, lowpass filter, highpass filter, lowpass filter.

\section{MRI Texture Analysis}

For the selected the 3 most useful features in the AP and HBP images, respectively, there was a statistically significant difference in the values of the 6 selected features by univariate analysis (Table 3). Variables significantly associated with VETC-positive HCC by univariate analysis, including clinical factors and texture features, were considered as candidates for backward stepwise multivariate

Table 4 Identified Performances of the Two Significant Variables and Combination for Predicting VETC-Positive HCC

\begin{tabular}{|l|c|c|c|c|c|c|}
\hline Variables & AUC & SEN (\%) & SPE (\%) & ACC (\%) & PLR & NLR \\
\hline AP image texture feature & & & & \\
\hline Log-sigma-4.0-mm-3D-first-order Kurtosis & $\begin{array}{c}0.808 \\
(0.731,0.886)\end{array}$ & $\begin{array}{c}71.3 \\
(58.9,83.6)\end{array}$ & $\begin{array}{c}81.5 \\
(73.0,89.9)\end{array}$ & $\begin{array}{c}77.4 \\
(77.2,77.7)\end{array}$ & $\begin{array}{c}3.842 \\
(2.357,6.262)\end{array}$ & $\begin{array}{c}0.354 \\
(0.228,0.549)\end{array}$ \\
\hline HBP image texture feature & $\begin{array}{c}0.774 \\
(0.695,0.853)\end{array}$ & $\begin{array}{c}92.3 \\
(85.1,99.6)\end{array}$ & $\begin{array}{c}49.4 \\
(38.5,60.3)\end{array}$ & $\begin{array}{c}66.2 \\
(65.8,66.5)\end{array}$ & $\begin{array}{c}1.824 \\
(1.450,2.293)\end{array}$ & $\begin{array}{c}0.156 \\
(0.059,0.410)\end{array}$ \\
\hline $\begin{array}{l}\text { Wavelet-LHL-GLDM } \\
\text { Dependence Non Uniformity Normalized }\end{array}$ & $\begin{array}{c}0.844 \\
(0.777,0.910)\end{array}$ & $\begin{array}{c}80.8 \\
(70.1,91.5)\end{array}$ & $\begin{array}{c}74.1 \\
(64.5,83.6)\end{array}$ & $\begin{array}{c}76.7 \\
(76.4,77.0)\end{array}$ & $\begin{array}{c}3.115 \\
(2.107,4.607)\end{array}$ & $\begin{array}{c}0.260 \\
(0.147,0.460)\end{array}$ \\
\hline Combination a
\end{tabular}

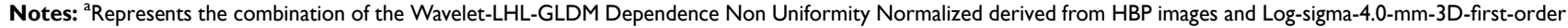
Kurtosis derived from AP images.

Abbreviations: AP, arterial phase; HBP, hepatobiliary phase; GLDM; gray level dependence matrix; LHL, lowpass filter, highpass filter, lowpass filter; ACC, accuracy; SEN, sensitivity; SPE, specificity; PLR, positive likelihood ratio; NPV, negative likelihood ratio. 


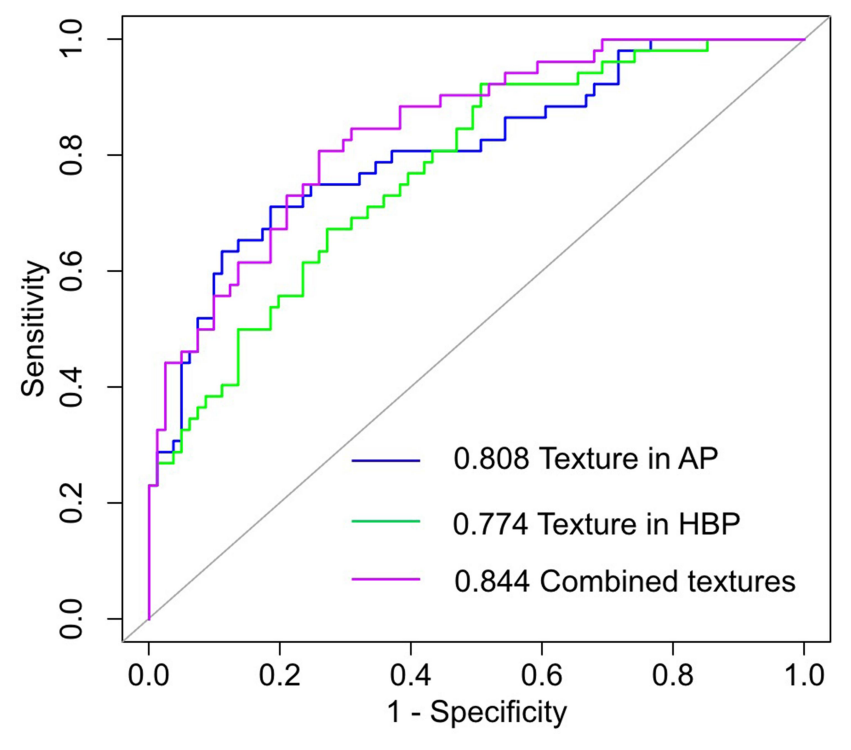

Figure 2 ROC for the two significant variables and the combination. Combined features represent the combination of the Log-sigma-4.0-mm-3D first-order Kurtosis derived from AP image and Wavelet-LHL-GLDM Dependence Non Uniformity Normalized derived from HBP images. Texture in AP represents the Log-sigma-4.0-mm-3D first-order Kurtosis derived from AP images. Texture in HBP represents the Wavelet-LHL-GLDM Dependence Non Uniformity Normalized derived from HBP images.

Notes: AP, arterial phase; HBP, hepatobiliary phase; LHL, lowpass filter, highpass filter, lowpass filter; GLDM, gray level dependence matrix.

analysis. In multivariate analysis, the Log-sigma-4.0-mm3D first-order Kurtosis derived from AP images (odds ratio $[\mathrm{OR}]=4.128, P=0.001)$ and the Wavelet-LHLGLDM Dependence Non Uniformity Normalized derived from HBP images $(\mathrm{OR}=2.280, P=0.004)$ were independent significant variables associated with VETC-positive HCC (Table 3), though the univariate analysis revealed that serum AFP level, serum AST level, and maximum diameter were associated with the VETC-positive HCC. Hosmer-Lemeshow test (Hosmer-Lemeshow Wald chisquare $=1.045, d f=3, P=0.790$ ) showed the goodness of fit for the combined two features to identify VETCpositive/negative $\mathrm{HCC}$.

The identified performance of these two selected texture features in multivariate analysis and the combination of the two features for identifying VETC-positive/negative HCC was shown in Table 4 and Figure 2. The combination of the two texture features had an AUC value of 0.844 (95\% CI, $0.777,0.910)$ with a sensitivity of $80.8 \%$, a specificity of $74.1 \%$, and an accuracy of $76.7 \%$ (Table 4 ). There was no statistically significant difference in AUC values (AUC = $0.808,0.774$ ) between the Log-sigma-4.0-mm-3D first-order Kurtosis derived from AP images and the Wavelet-LHLGLDM Dependence Non Uniformity Normalized derived from HBP images $(\mathrm{z}=0.723, P=0.470)$ (Table 5). The combination of the two texture features showed a better identified performance than the Wavelet-LHL-GLDM Dependence Non Uniformity Normalized derived from HBP image $(\mathrm{z}=-2.406, P=0.016)$, while no significantly better than the Log-sigma-4.0-mm-3D first-order Kurtosis derived from AP image only $(\mathrm{z}=-1.523, P=0.128)$ (Table $5)$. The optimal cut-off values for sensitivity and specificity in predicting VETC-positive HCC were 3.415 for The Logsigma-4.0-mm-3D first-order Kurtosis derived from AP images, 0.058 for the Wavelet-LHL-GLDM Dependence Non Uniformity Normalized derived from HBP images (Table 5). The representative examples to identify VETCpositive/negative HCC by texture analysis of Gd-EOBDTPA-enhanced MRI were shown in Figures 3 and 4.

Table 5 Comparison of the Identified Performances of Significant Variables

\begin{tabular}{|c|c|c|c|c|}
\hline Variables & Cut-Off Value & AUC & $P^{b}$ value & $P^{c}$ value \\
\hline \multicolumn{5}{|l|}{ AP image texture feature } \\
\hline $\begin{array}{l}\text { Log-sigma-4.0-mm-3D- } \\
\text { first-order Kurtosis }\end{array}$ & 3.415 & $\begin{array}{c}0.808 \\
(0.731,0.886)\end{array}$ & & 0.470 \\
\hline \multicolumn{5}{|l|}{ HBP image texture feature } \\
\hline $\begin{array}{l}\text { Wavelet-LHL-GLDM } \\
\text { Dependence Non Uniformity Normalized }\end{array}$ & 0.058 & $\begin{array}{c}0.774 \\
(0.695,0.853)\end{array}$ & 0.470 & \\
\hline Combination $^{a}$ & & $\begin{array}{c}0.844 \\
(0.777,0.910)\end{array}$ & 0.128 & 0.016 \\
\hline
\end{tabular}

Notes: ${ }^{a}$ Represents the combination of the Wavelet-LHL-GLDM Dependence Non Uniformity Normalized derived from HBP images and Log-sigma-4.0-mm-3D-first-order Kurtosis derived from AP images. ${ }^{b}$ The $P$ value represents the difference in AUC values compared with the Log-sigma-4.0-mm-3D-first-order Kurtosis derived from AP images. ${ }^{C}$ The $P$ value represents the difference in AUC values compared with the Wavelet-LHL-GLDM Dependence Non Uniformity Normalized derived from HBP images.

Abbreviations: 3D, three dimensions; GLDM, gray level dependence matrix. LHL, lowpass filter, highpass filter, lowpass filter. 

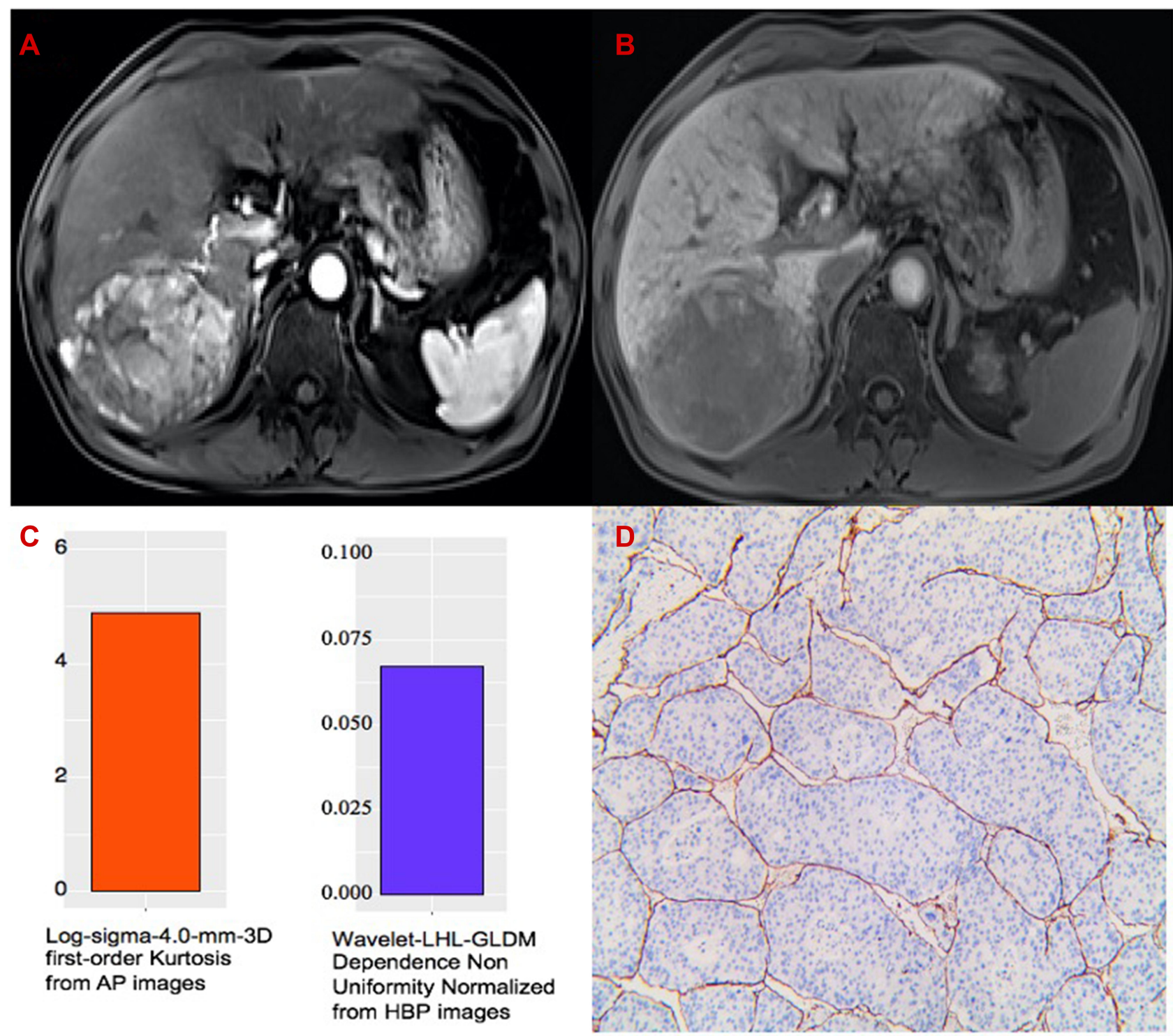

Figure 3 The representative example to identify VETC-positive HCC by texture analysis of Gd-EOB-DTPA-enhanced MRI. (A) AP image. (B) HBP image. (C) The values of 4.898 and 0.067 of the two textures are greater than their cut-off values. (D) Immunohistochemical staining for CD34 (original magnifications $x$ I00). VETC-positive HCC shows clusters of tumor cells bordered by a complete rim of CD34-positive endothelial cells in more than $5 \%$ of the tumor area.

Abbreviations: AP, arterial phase; HBP, hepatobiliary phase; LHL, lowpass filter, highpass filter, lowpass filter; GLDM, gray level dependence matrix.

\section{Discussion}

The VETC, a histological vascular pattern, has been identified as a predictor of micro-metastasis, ${ }^{9}$ aggressive and poorer prognosis in HCC. ${ }^{9,14}$ Our study demonstrated that texture analysis based on Gd-EOB-DTPA-enhanced MRI was superior to clinical factors for the identification of VETC status of HCC. The Log-sigma-4.0-mm-3D firstorder Kurtosis derived from AP image and Wavelet-LHLGLDM Dependence Non Uniformity Normalized derived from HBP images were independent variables for identifying VETC-positive HCC. The combination of the two texture features achieved good identified performance with an AUC value of 0.844 (95\% CI, 0.777-0.910). Additionally, the Log-sigma-4.0-mm-3D first-order Kurtosis derived from AP images showed comparable diagnostic performance with an AUC value of 0.808 ( $95 \%$ CI, $0.731,0.886)$. These findings suggest that texture features based on Gd-EOB-DTPA-enhanced MRI can reflect the complex internal structure of VETC-positive /negative HCC.

In our study, we selected AP and HBP images based on Gd-EOB-DTPA-enhanced MRI because AP is characterized 


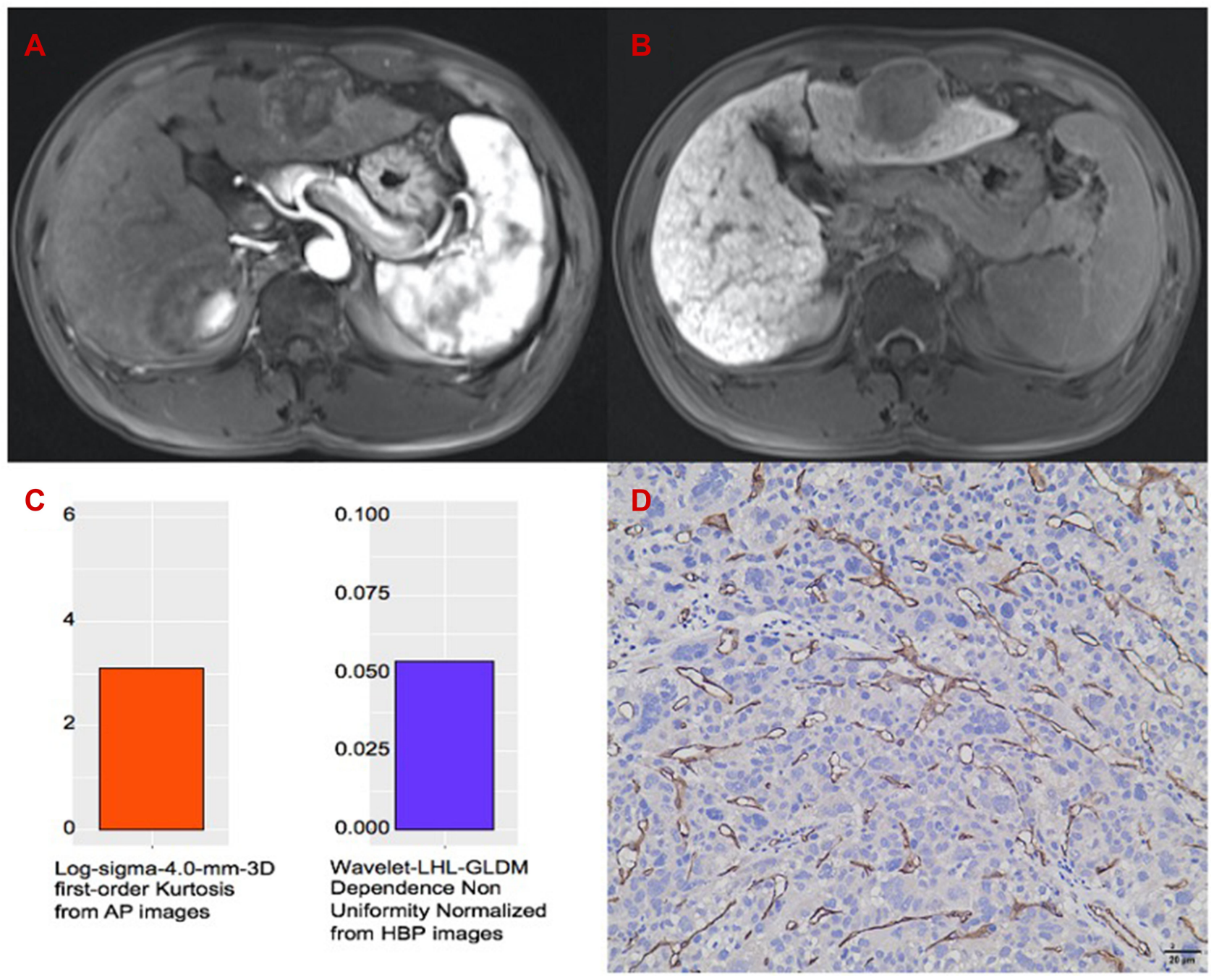

Figure 4 The representative example to identify VETC-negative HCC by texture analysis of Gd-EOB-DTPA-enhanced MRI. (A) AP image. (B) HBP image. (C) The values of 3.110 and 0.054 of the two textures are lower than their cut-off values. (D) Immunohistochemical staining for CD34 (Original magnifications $\times 200$ ). VETC-negative HCC was absent of vessels that formed cobweb-like networks with an unequivocal and continuous lining of CD34 positive endothelium.

Abbreviations: AP, arterial phase; HBP, hepatobiliary phase; LHL, lowpass filter, highpass filter, lowpass filter; GLDM, gray level dependence matrix.

by lesion enhancement mediated by vascular changes in HCC and HBP by hepatocyte uptake of contrast agent. According to the definitions of the features derived from the pyradiomics library and the IBSI, ${ }^{26,29}$ the first-order Kurtosis is a measure of the 'peakedness' of the distribution of values in the image ROI. A higher kurtosis reflects that the mass of the distribution is concentrated towards the tail(s) rather than towards the mean; the GLDM Dependence Non Uniformity Normalized measures the similarity of dependence throughout the image, with a higher value indicating more heterogeneity among dependencies in the image. Our results showed that VETCpositive HCCs had higher values of Kurtosis and Dependence Non Uniformity Normalized than VETCnegative HCCs, which was related to pathological characteristics of VETC-positive/negative HCCs. Our results were also consistent with previous studies in which VETC has been identified as a vascular pattern composed of functional vessels with blood perfusion, and an arterial phase hypovascular component and substantial necrosis presented on MRI were associated with macrotrabecularmassive $\mathrm{HCC}$, which have similar pathological figures to partial VETC-positive HCC. ${ }^{8,19,20}$ Accordingly, the pixel intensities of ROI indicated by Kurtosis was higher in VETC-positive HCCs than VETC-positive HCCs. Additionally, VETC-positive HCCs had more diverse vascular patterns, including VETC, sinusoidal capillarization/ incomplete angiogenesis, ${ }^{30}$ and other neovascularization patterns, which may result in additional heterogeneity of the texture compared with VETC-negative HCCs. 
Conventional lesion intensity and heterogeneity were judged by a subjective assessment of radiologists, which could vary depending on individual experience level and personal preference; however, texture analysis allowed an objective assessment of lesion intensity and heterogeneity. Indeed, our group has revealed that the AUC values of textures derived from AP and HBP images were significantly higher than that of qualitative characteristics and semi-quantitative characteristics (ratio of tumor to liver signal intensity) in AP and HBP images respectively, compared with our previous study. ${ }^{18}$

Our study has several limitations. First, selection bias was inevitable because of the retrospective design. Second, we defined "VETC $\geq 5 \%$ " as the VETC-positive group in this study, referring to previous studies. ${ }^{8-10}$ However, the optimal cut-off value of VETC status is not yet standardized in patients with HCC, as the VETC is a novel vascular pattern, which has not been widely investigated. Third, we only used AP and HBP images of Gd-EOB-DTPA-enhanced MRI in this study; thus, the comparison with pre-contrast, T2W, PVP, and TP images was lacking. Fourth, this study was conducted in a single institution, findings of which have not been validated by external data. Then, multicenter and prospective studies are needed to validate the texture analysis of VETC-positive/negative HCCs as a clinical tool.

\section{Conclusions}

The Log-sigma-4.0-mm-3D first-order Kurtosis derived from AP images and the Wavelet-LHL-GLDM Dependence Non Uniformity Normalized derived from HBP image can reflect the complex internal structure of VETC-positive/negative HCC and help identify VETC-positive HCC. Then, pretreatment identifying VETC status could help select HCC patients with poor prognosis, early recurrence, and Sorafenib benefit. Therefore, it can provide valuable information for determining treatment decision-making.

\section{Abbreviations}

AP, Arterial phase; HBP, hepatobiliary phase; GLCM, gray level co-occurrence matrix; GLDM; gray level dependence matrix; GLRLM, gray level run length matrix; GLSZM, gray level size zone matrix; NGTDM, neighboring gray tone dependence matrix.

\section{Data Sharing Statement}

The datasets used or analyzed during the current study available from the corresponding author on reasonable request.

\section{Consent for Publication}

Not applicable.

\section{Ethics Approval and Informed Consent}

The Institutional Review Board (IRB) at the First Affiliated Hospital of Soochow University approved this retrospective study. The informed consent from patient was waived because of the retrospective nature of the study.

\section{Author Contributions}

All authors made a significant contribution to the work reported, whether that is in the conception, study design, execution, acquisition of data, analysis and interpretation, or in all these areas; took part in drafting, revising or critically reviewing the article; gave final approval of the version to be published; have agreed on the journal to which the article has been submitted; and agree to be accountable for all aspects of the work.

\section{Funding}

This work has been supported by the National Natural Science Foundation of China (81801692), Suzhou Municipal Science and Technology Bureau (SYS2020125, SS201808) and National Key Research and Development Program of China (2017YFC0114300).

\section{Disclosure}

The authors declare that they have no competing interests.

\section{References}

1. Bray F, Ferlay J, Soerjomataram I, et al. Global cancer statistics 2018: GLOBOCAN estimates of incidence and mortality worldwide for 36 cancers in 185 countries. CA Cancer J Clin. 2018;68:394-424.

2. Llovet JM, Montal R, Sia D, Finn RS. Molecular therapies and precision medicine for hepatocellular carcinoma. Nat Rev Clin Oncol. 2018;15:599-616.

3. Galle PR, Forner A, Llovet JM, et al. EASL clinical practice guidelines: management of hepatocellular carcinoma (vol 69, pg 182, 2018). $J$ Hepatol. 2019;70:817.

4. Marrero JA, Kulik LM, Sirlin CB, et al. Diagnosis, staging, and management of hepatocellular carcinoma: 2018 practice guidance by the American association for the study of liver diseases. Hepatology. 2018;68:723-750.

5. Yang JD, Heimbach JK. New advances in the diagnosis and management of hepatocellular carcinoma. BMJ. 2020;371:m3544.

6. Tabrizian P, Jibara G, Shrager B, et al. Recurrence of hepatocellular cancer after resection patterns, treatments, and prognosis. Ann Surg. 2015;261:947-955.

7. Roayaie S, Obeidat K, Sposito C, et al. Resection of hepatocellular cancer $\leq 2 \mathrm{~cm}$ : results from two Western centers. Hepatology. 2013;57:1426-1435. 
8. Fang J-H, Zhou H-C, Zhang C, et al. A novel vascular pattern promotes metastasis of hepatocellular carcinoma in an epithelial-mesenchymal transition-independent manner. Hepatology. 2015;62:452-465.

9. Ding T, Xu J, Zhang Y, et al. Endothelium-coated tumor clusters are associated with poor prognosis and micrometastasis of hepatocellular carcinoma after resection. Cancer. 2011;117:4878-4889.

10. Renne SL, Woo HY, Allegra S, et al. Vessels Encapsulating Tumor Clusters (VETC) . is a powerful predictor of aggressive hepatocellular carcinomaHepatology. 2020;71:183-195.

11. Sugino T, Yamaguchi T, Ogura G, et al. Morphological evidence for an invasion-independent metastasis pathway exists in multiple human cancers. BMC Med. 2004;2.

12. Sugino T, Yamaguchi T, Hoshi N, et al. Sinusoidal tumor angiogenesis is a key component in hepatocellular carcinoma metastasis. Clin Exp Metastasis. 2008;25:835-841.

13. Zhou HC, Fang JH, Shang LR, et al. MicroRNAs miR-125b and miR-100 suppress metastasis of hepatocellular carcinoma by disrupting the formation of vessels that encapsulate tumour clusters J Pathol. 2016;240:450-460.

14. Fang J-H, Xu L, Shang L-R, et al. Vessels that Encapsulate Tumor Clusters (VETC) pattern is a predictor of sorafenib benefit in patients with hepatocellular carcinoma. Hepatology. 2019;70:824-839.

15. Zhou L, Rui JA, Zhou WX, et al. Edmondson-Steiner grade: a crucial predictor of recurrence and survival in hepatocellular carcinoma without microvascular invasio. Pathol Res Pract. 2017;213:824-830.

16. Xu X, Zhang H-L, Liu Q-P, et al. Radiomic analysis of contrast-enhanced $\mathrm{CT}$ predicts microvascular invasion and outcome in hepatocellular carcinoma. J Hepatol. 2019;70:1133-1144.

17. Lim KC, Chow PK, Allen JC, et al. Microvascular invasion is a better predictor of tumor recurrence and overall survival following surgical resection for hepatocellular carcinoma compared to the Milan criteria. Ann Surg. 2011;254:108-113.

18. Fan Y, Yu Y, Hu M, et al. Imaging features based on Gd-EOB-DTPAenhanced MRI for predicting vessels encapsulating tumor clusters (VETC) in patients with hepatocellular carcinoma. $\mathrm{Br} J$ Radiol. 2021;94:20200950.

19. Rhee H, Cho ES, Nahm JH, et al. Gadoxetic acid-enhanced MRI of macrotrabecular-massive hepatocellular carcinoma and its prognostic implications. J Hepatol. 2021;74:109-121.
20. Mulé S, Galletto Pregliasco A, Tenenhaus A, et al. Multiphase liver MRI for identifying the macrotrabecular-massive subtype of Hepatocellular carcinoma. Radiology. 2020;295:562-571.

21. Wang HQ, Yang C, Zeng MS, et al. Magnetic resonance texture analysis for the identification of cytokeratin 19-positive hepatocellular carcinoma. Eur J Radiol. 2019;117:164-170.

22. Tabari A, Torriani M, Miller KK, et al. Anorexia nervosa: analysis of trabecular texture with CT. Radiology. 2017;283:178-185.

23. Önner H, Abdülrezzak Ü, Tutuş A. Could the skewness and kurtosis texture parameters of lesions obtained from pretreatment Ga-68 DOTA-TATE PET/CT images predict receptor radionuclide therapy response in patients with gastroenteropancreatic neuroendocrine tumors? Nucl Med Commun. 2020;41:1034-1039.

24. van Griethuysen JJM, Fedorov A, Parmar C, et al. Computational radiomics system to decode the radiographic phenotype. Cancer Res. 2017;77:e104-e107.

25. Schwier M, van Griethuysen J, Vangel MG, et al. Repeatability of multiparametric prostate MRI radiomics features. Sci Rep. 2019;9:9441.

26. Zwanenburg A, Vallières M, Abdalah MA, et al. The image biomarker standardization initiative: standardized quantitative radiomics for high-throughput image-based phenotyping. Radiology. 2020;295:328-338.

27. Wang W, Gu D, Wei J, et al. A radiomics-based biomarker for cytokeratin 19 status of hepatocellular carcinoma with gadoxetic acid-enhanced MRI. Eur Radiol. 2020;30:3004-3014.

28. Vasquez MM, Hu C, Roe DJ, et al. Least absolute shrinkage and selection operator type methods for the identification of serum biomarkers of overweight and obesity: simulation and application. $B M C$ Med Res Methodol. 2016;16.

29. Pyradiomics. Radiomics features. Available from: https://pyradio mics.readthedocs.io/en/lastest/feature.html. Accessed April 7, 2021.

30. Sciarra A, Di Tommaso L, Nakano M, et al. Morphophenotypic changes in human multistep hepatocarcinogenesis with translational implications. J Hepatol. 2016;64:87-93.

\section{Publish your work in this journal}

The Journal of Hepatocellular Carcinoma is an international, peerreviewed, open access journal that offers a platform for the dissemination and study of clinical, translational and basic research findings in this rapidly developing field. Development in areas including, but not limited to, epidemiology, vaccination, hepatitis therapy, pathology and molecular tumor classification and prognostication are all considered for publication. The manuscript management system is completely online and includes a very quick and fair peer-review system, which is all easy to use. Visit http://www.dovepress.com/ testimonials.php to read real quotes from published authors 